\title{
Guidance on conducting 2D linear viscoelastic site response analysis using a finite element code
}

\author{
Carolina Volpini ${ }^{1}$, John Douglas ${ }^{1}$ and Andreas Hvidtfelt Nielsen ${ }^{2}$ \\ 1- Department of Civil and Environmental Engineering, University of Strathclyde, Glasgow \\ $(U K)$
}

2- Atkins (member of the SNC-Lavalin Group), Glasgow (UK)

Corresponding author e-mail: carolina.volpini@ strath.ac.uk

\begin{abstract}
Various software packages are available to conduct one-dimensional (1D) and twodimensional (2D) site response analyses (SRAs). In this article, a finite element program is tested with the purpose of assessing the importance of several aspects on the obtained results and verifying the software. Abaqus (Dassault Systèmes) is compared with the 1D SRA software STRATA (Kottke \& Rathje, 2008) for simple 1D models to understand the influence of the boundary conditions; as being a 1D SRA program, STRATA does not require vertical boundary conditions. For Abaqus the subroutine by Nielsen $(2006,2014)$ is used to implement free-field boundary conditions.

In addition, we test the influence of mesh dimension and Rayleigh damping as well as the importance of buffer-zone width. 2D SRAs with Abaqus and FLAC3D (Itasca Consulting Group, 2012), which is commonly used for geotechnical analyses, are compared as part of the assessment. Similar results are obtained from the two programs but Abaqus is preferred as it is more efficient for linear elastic analyses than FLAC3D, which, on the contrary, performs well for soil presenting strongly non-linear behaviour and effective stress. We demonstrate that reliable results can be achieved, not only for simple uniform sites but also for complex sites with multiple layers and dipping stratigraphy.
\end{abstract}

\section{Keywords}

One-dimensional, two-dimensional, site response analysis, free-field boundary conditions, damping 


\section{Introduction}

An important part of geotechnical earthquake engineering is the study of the response of the ground under earthquake excitation by means of site response analysis (SRA). Based on the characteristics of the problem studied, several approaches can be used: from one-dimensional (1D) to threedimensional (3D) analyses and from linear-elastic to fully non-linear soil behaviour. 1D linearelastic SRA is often a good starting point for all such studies, even though it is unrealistic in many cases (e.g. when the site is subject to high-amplitude shaking, for which a non-linear analysis would provide more accurate results, and in the case of sedimentary valleys and basins, where 3D effects are pronounced). A complete SRA should include a consideration of the 3D geometry and nonlinear soil behaviour and the consideration of effective stress, which is important, for example, for the study of soil liquefaction. The seismic waves, under these conditions, can generate an increase in the pore water pressure producing a reduction of stiffness and strength of the soil. However, it is not common to perform such analyses, because the more complex a model is, the more input parameters are needed, which means that a full characterization of the site must be available to the engineer. In addition, 3D and non-linear analyses require long computational times, sophisticated software and much experience in conducting the analyses and in interpreting the results.

In this work, we test several approaches, but we will limit ourselves to 1D and 2D SRA and to linear-elastic behaviour with viscous damping. A recent example of a comparison between 1D and 2D SRA can be found in Volpini and Douglas (2018). We make several comparisons between different software packages and test their capabilities to treat irregular geometries, such as gently dipping layers. We use a finite element (FE) code, Abaqus (Dassault Systèmes), and a finite difference code, FLAC3D (Itasca Consulting Group, 2012), for the 2D analyses, and the code STRATA (Kottke and Rathje, 2008) for the 1D simulations. We then examine the shape of the amplification functions obtained from different software packages as they yield valuable physical insight into the frequency dependence of the response. 


\section{One-Dimensional Approach}

The easiest way to conduct a SRA is to use a 1D approach, where the soil deposits are modelled as a layered column. The first popular program developed for such analyses was SHAKE (Schnabel et al, 1972). Following SHAKE, several other software packages have been published using the same basic approach including STRATA. In this section, some of the basic analytical results for 1D SRA are presented as they are useful for the rest of the article.

The 1D approach is underpinned by two fundamental assumptions: (1) the ground is composed of one or more parallel layers, extending to infinity in the horizontal plane; and (2) incoming seismic waves follow vertical ray paths. This final assumption is normally justified through Snell's law, which informs us that inclined waves propagating through horizontal layers of soil layers with successively lower impedances will be refracted closer to a vertical path (Kramer, 1996).

The simplest case is a single layer of soil overlying an infinite nearly-rigid half-space. Assuming linear-elastic material properties, the amplification function $A_{r}(\omega)$, which is defined as the ratio between the displacement amplitude recorded at the surface and the amplitude of the input motion entering at the base of the layer, can be computed as (Kramer, 1996):

$$
A_{r}(\omega)=\left|H_{r}(\omega)\right|=\left|\frac{U(0, \omega)}{U(H, \omega)}\right|=\frac{1}{\left|\cos \left(\frac{\omega H}{V_{s}}\right)\right|} \geq 1
$$

where $H_{r}(\omega)$ is the complex transfer function, $U(z, \omega)$ is the magnitude of horizontal displacement at depth $z, \omega$ is angular frequency, and $H$ and $V_{s}$ are the depth and shear-wave velocity of the soil layer, respectively. For $\frac{\omega H}{V_{s}}=\frac{\pi}{2}+n \pi$, with $n=0,1,2, \ldots$, the amplification function tends to infinity, which signifies resonance. For $n=0, \omega$ is equal to the natural or fundamental frequency of the layer, which is given by: 


$$
\omega_{r}=\frac{\pi V_{s}}{2 H} \quad, \quad f_{r}=\frac{V_{s}}{4 H}
$$

The amplification function can be modified to consider the effect of energy dissipation (damping) within the soil. The simplest approach is to assume that the material damping is of the viscous type. Incorporating damping means the displacement amplitudes associated with the resonant frequencies are no longer infinite, and equation (1) is modified to (Kramer, 1996):

$$
\begin{aligned}
& A_{r}^{*}(\omega)=\left|H_{r}{ }^{*}(\omega)\right|=\frac{1}{\sqrt{\cos ^{2}(k H)+(D k H)^{2}}} \\
& \left(A_{r}^{*}\right)_{\max } \cong \frac{2}{(2 n-1) \pi D} \quad n=1, \ldots, \infty
\end{aligned}
$$

where $k$ is the wave number and $D$ is the damping ratio.

In this article, only linear-elastic models with viscous damping are considered. It is possible to model the non-linear behaviour of the soil, which is more realistic especially for high-amplitude shaking. In practice, the equivalent linear approach (for low to moderate levels of soil nonlinearity) is often used (Schnabel et al, 1991), in which the shear modulus and damping ratio of each layer are adjusted after each analysis, based on the peak shear strain observed during the analysis. The analysis is then repeated until the properties converge to a stable set of values. The other option is a fully non-linear approach (Bonilla, 2000). Comparisons between equivalent linear analysis and fully non-linear analysis are presented by Kaklamanos et al. (2013, 2015). Kaklamanos et al. (2013) suggest limits, in terms of maximum shear strain and peak ground acceleration (PGA), of when linear and equivalent-linear analyses are valid.

The 1D approach is no longer valid for sites characterised by irregular subsurface stratigraphy, irregular surface topography or incident body waves with non-vertical ray paths (or incident surface waves). A typical example is a sedimentary valley, where the soil deposit is much softer that the 
bedrock; in such conditions, a complex wave field can be generated due to multiple refractions and reflections in the interfaces, which can lead to long duration ground motions and high amplification. Indeed, the soil deposits overlying the curvature of a basin can trap body waves and convert body waves into standing surface waves (Vidale et al, 1988; Bard and Bouchon, 1980a, 1980b; Bard and Bouchon, 1985; Chávez-García and Faccioli, 2000). This kind of situation requires 2D or ideally 3D SRA. Useful studies on the applicability of 1D SRA for different situations are by Thompson et al. (2012), Stewart et al. (2014) and Stewart et al. (2017).

\section{Two-Dimensional Approach}

\subsection{Limitations of the 2D approach}

As previously mentioned, when a real site is characterised by irregular subsurface stratigraphy and/or surface topography, it is necessary to use 2D or 3D SRA. The jump from one dimension to multiple dimensions involves additional challenges. First, it must be noted that it is impossible to construct an equivalent 2D slice that matches the dynamic behaviour of a true $3 \mathrm{D}$ site. A $2 \mathrm{D}$ model with the same dimensions and material properties will generally overestimate the soil's dynamic stiffness and radiation damping due to geometric spreading of waves (Wolf, 1994). Nevertheless, the study of $2 \mathrm{D}$ models is instructive as they pose many of the same practical challenges as $3 \mathrm{D}$ models. In this section we outline some of the key considerations.

\subsection{Boundary conditions}

An earthquake affects a large area of the Earth's surface. However, engineers are usually concerned with the response of a relatively small site and a single structure. Instead of modelling the entire region affected by an earthquake, which is generally not feasible and almost never desirable, it is common practice to truncate the model at a certain distance from the region of interest. This truncation introduces artificial borders around the model. The geological media outside these artificial borders are assumed to be unbounded (semi-infinite), and their presence is 
simulated by enforcement of appropriate boundary conditions. The literature on this subject is vast. However, it is possible to distinguish between two fundamental types of boundary conditions: elementary boundary conditions (EBCs) and absorbing boundary conditions (ABCs).

The EBCs are either fixed or free. With EBCs, the numerical model should be so large that any waves reflected at the boundaries do not have time to return to the central region of the model. Much smaller models are feasible with ABCs, which therefore have gained much interest.

ABCs can be either global or local. In a global scheme, each boundary node is fully coupled to all other boundary nodes in both space and time. In a local scheme, the solution at any time step depends only on the current node and the current time step, and perhaps a few neighbouring points in time and space. Theoretically speaking, global boundaries are exact (although exact solutions are rarely attained in practice). Local boundaries are approximate, but appear much more attractive for numerical implementation than global boundaries.

The first local ABC was proposed by Lysmer and Kuhlemeyer (1969), who used viscous boundary tractions (dashpots) to absorb outgoing waves. For a vertical boundary parallel with the $y$-axis, the tractions can be written as:

$$
\begin{aligned}
& f_{x}=-\rho V_{p} \frac{\partial u}{\partial t} \\
& f_{y}=-\rho V_{s} \frac{\partial v}{\partial t}
\end{aligned}
$$

where $\rho$ is the material density, $V_{p}$ is the $\mathrm{P}$-wave speed, $V_{s}$ is the $\mathrm{S}$-wave speed, and $(u, v)$ are displacements in the $(x, y)$ directions, respectively. This boundary condition is completely effective at absorbing body waves approaching the boundary at normal incidence. For oblique angles of incidence and for surface waves there is still energy absorption, but it is not perfect. The viscous boundary is currently the only type of $\mathrm{ABC}$ available in Abaqus. 
The viscous boundary works well when the seismic source is within the model, but when the seismic waves enter through the base of the model, an extension is required. The problem is that the viscous boundary defined on the vertical boundaries of the model will attenuate the incoming seismic waves as they travel up through the model. A solution is to define the dashpots such that they act on the scattered waves propagating outwards from the centre of the model. The scattered wave field may be computed as the difference between the wave field that would exist in the absence of any irregularities within the main model (also called the free-field motion) and the total motion at the boundaries of the model. This solution is often called free-field boundary conditions. The free-field boundary conditions were first introduced by Wolf (1988) and Zienkiewicz et al. (1989). The solution requires an independent free-field model as an extension to the main model (Figure 1). In the 2D case, the free-field model comprises two soil columns placed at the edges of the main model, with each column acting as a 1D model. In practice, this extension requires the analysis of free-field motions either prior to, or in parallel with, the analysis of the main model. The main model and the free-field columns are coupled through the normal and shear tractions, $f_{x}$ and $f_{y}$, defined as:

$$
\begin{aligned}
& f_{x}=\rho V_{p}\left(\frac{\partial u^{\prime}}{\partial t}-\frac{\partial u}{\partial t}\right)+\ell_{x} \sigma_{x}^{\prime} \\
& f_{y}=\rho V_{s}\left(\frac{\partial v^{\prime}}{\partial t}-\frac{\partial v}{d t}\right)+\ell_{x} \tau_{x y}^{\prime}
\end{aligned}
$$

where prime indicates a quantity evaluated in the free-field, $\ell_{x}$ equals +1 if an outward normal points in the positive $x$ direction and equals -1 if it points in the negative $x$ direction. The first part of the equation is the viscous boundary as per equation (4). The second term of the equation is the surface stress required to maintain free-field wave propagation plus any static reactions. In this manner, the viscous boundary absorbs only scattered waves due to irregularities within the main model, but leaves the input motion unaffected. 
We use the free-field boundary conditions in this work, both in Abaqus and in FLAC3D. The procedure is not directly available in Abaqus; however, Nielsen $(2006,2014)$ has shown how it may be implemented, and we follow this procedure.

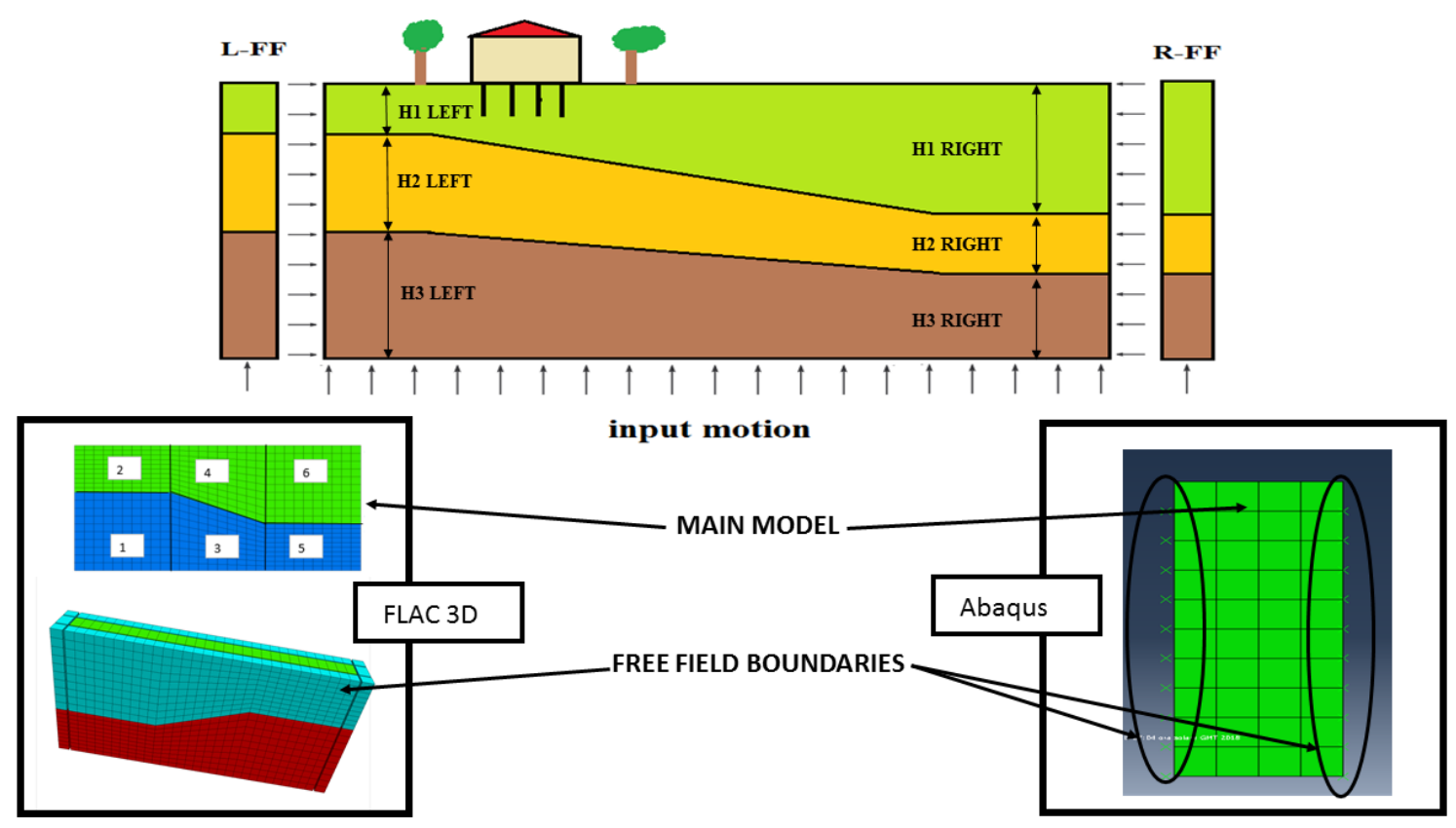

Figure 1. Example showing how both FLAC3D and Abaqus display free-field boundaries

\subsection{Mesh Dimension}

When modelling wave propagation within a continuum in $2 \mathrm{D}$ or $3 \mathrm{D}$, it is important how the space is discretized. In contrast to the 1D approach, where no dimensions except for the height of the column are considered, there is also a width in $2 \mathrm{D}$ (and breadth for $3 \mathrm{D}$ domains). According to Kuhlemeyer and Lysmer (1973), the maximum frequency transmitted by a model can be estimated based on the largest elements or zones within the slowest material as follows:

$$
\Delta l_{\max } \leq \frac{\lambda_{\min }}{10} \leq \frac{V_{s, \min }}{10 f_{\max }} \quad \text { or } \quad f_{\max } \leq \frac{V_{s, \min }}{10 \Delta \lambda_{\max }}
$$

where $\Delta l_{\text {max }}$ is the maximum dimension of the element, $\lambda$ the wavelength of the passing wave, $V_{s}$ is the layer's shear-wave velocity and $f_{\max }$ is the maximum frequency of interest, which is typically 
around $10-15 \mathrm{~Hz}$.

\subsection{Input Motion}

The input motion can be an external or internal dynamic action. In this work, we consider just the external option, defined as an input motion applied to the base of the model, which can be flexible or rigid. If the base is flexible, the input motion can be applied by imposing a traction at the base of the model (option *DLOAD in Abaqus), which is the same as the approach of FLAC; whereas, if it is rigid, the motion is imposed as an acceleration or displacement time history. In this article we assume a rigid base, and we restrict our investigations to the case of horizontal base excitation. The input we are using is a within motion, which has been applied as an acceleration time-history in all three software packages (Abaqus, FLAC3D and STRATA). An example of the type of motion used is displayed in Figure 2.
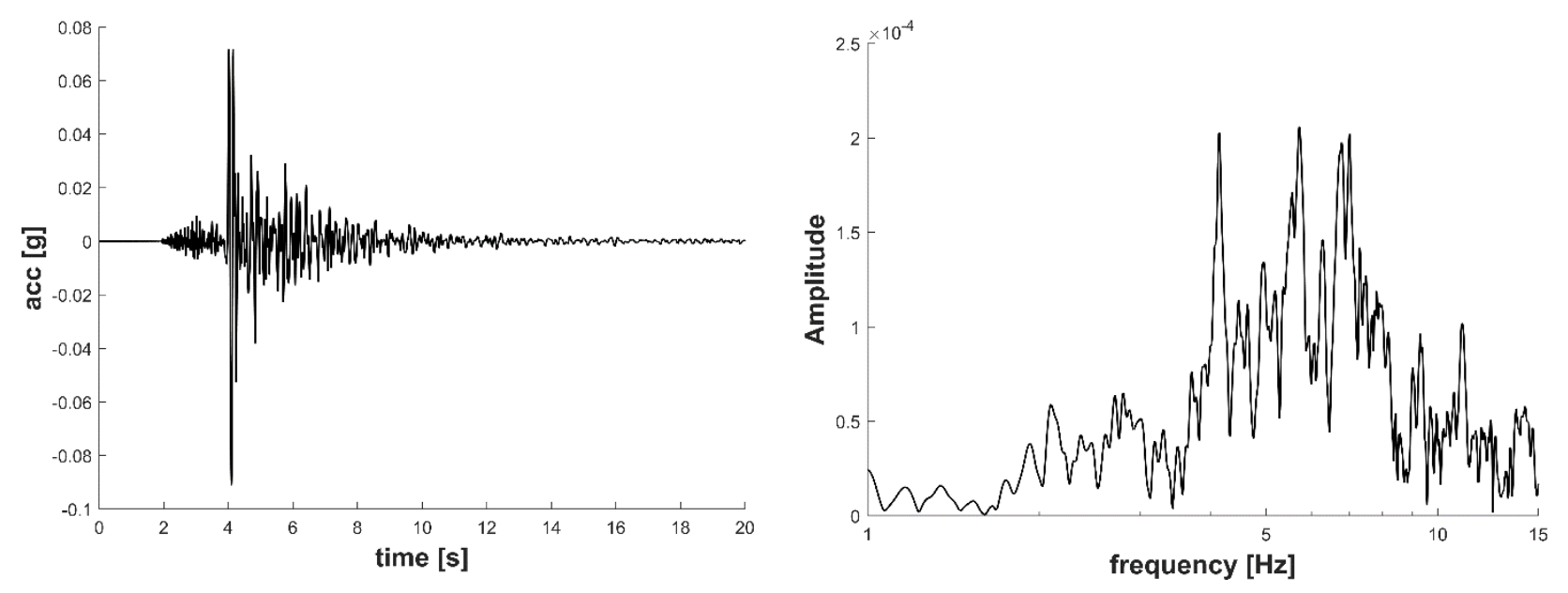

Figure 2. Example of input motion chosen from the ITACA database, PGA $=0.09 \mathrm{~g}$

(Luzi et al., 2017)

\subsection{Damping}

Damping, introduced in equation 7, 8 and 9, is a measure of the dissipative characteristics of the material and it has been found experimentally to be independent of frequency (Kimball and Lowell, 1927). For this reason, linear viscoelastic frequency-domain analysis (e.g. via STRATA) may be preferred over time-domain analysis (e.g. via Abaqus or FLAC3D) as it is easier to model 
frequency-independent damping in the former. In this study we are using and comparing the two approaches so an approximation must be found to simulate "constant" damping over the frequency range of interest.

Both Abaqus and FLAC3D allow the use of Rayleigh damping (Rayleigh and Lindsay, 1945), where the damping matrix is a linear combination of the mass and stiffness matrices using the coefficients $\alpha$ and $\beta$ :

$$
[C]=\alpha[M]+\beta[K]
$$

The damping ratio at frequency $\omega_{i}$ for a multiple degree-of-freedom system can be found using (Bathe and Wilson, 1976):

$$
\xi_{i}=\frac{1}{2}\left(\frac{\alpha}{\omega_{i}}+\beta \omega_{i}\right)
$$

A system of two equations is needed to determine $\alpha$ and $\beta$ from any two known frequencies, $\omega_{0}$ and $\omega_{1}$ :

$$
\alpha=\xi \frac{2 \omega_{0} 3 \omega_{1}}{\omega_{0}+\omega_{1}} \quad \text { and } \beta=\xi \frac{2}{\omega_{0}+\omega_{1}}
$$

The literature contains various suggestions about how to choose these frequencies. Chopra (1995) affirms the most common position that modes $n$ and $m$ should be specified to ensure reasonable damping values for all frequencies contributing to the response. Hashash and Park (2002) pointed out that for 1D linear SRA the two frequencies should represent the soil column's fundamental mode $\left(f_{0}\right)$ and one of its higher modes with a frequency given by multiplying $f_{0}$ by an odd integer (e.g. 3,5 or 7$)$.

Abaqus uses the complete equation (8), but FLAC3D uses a simplified method, where only a single 
frequency can be specified. This appears to be the frequency where the damping is lowest, which occurs when the derivative of the equation becomes zero, i.e.:

$$
\frac{d \xi}{d \omega}=0 \Rightarrow \omega_{\min }=\sqrt{\frac{\alpha}{\beta}} \quad \text { or } f_{\min }=\frac{1}{2 \pi} \sqrt{\frac{\alpha}{\beta}}
$$

The choice of $\omega_{\min }$ is not obvious. As the authors of FLAC suggest, once $\omega_{\min }$ is chosen, the damping ratio should be almost constant over a range between $\omega_{\min } / 1.5$ and $2 \omega_{\min }$. Thus, $\omega_{\min }$ should be chosen as a value lying in the centre of the range of frequencies present in the model or the predominant input frequencies.

\section{Testing Abaqus}

Abaqus is a multi-physics FE software package. Certain types of boundary conditions, such as freefield boundary conditions, are not standard options. When performing a 1D analysis (single column with flat layers), it is possible to use tied boundaries, where we constrain the motion of each node on the right boundary to the corresponding node on the left boundary or vice versa. Any pair of two nodes must be at the same level and must present the same stiffness, so this simplification is useful only for flat layers and not irregular models. To use free-field boundary conditions, it is necessary to extend Abaqus by means of the UEL (user subroutine to define an element) code written by Nielsen $(2006,2014)$ for implicit dynamic analysis.

As Abaqus is the focus of our study, it is useful to conduct several analyses to understand its capabilities. These tests aim to evaluate the:

- ability of the free-field subroutine to model the simple case of 1D wave propagation and a comparison to the solution with tied boundaries;

- influence of the mesh dimension; 
- difference between symmetric and asymmetric matrices for the free-field boundary option;

- influence of the choice of frequency for the damping formulation; and

- influence of the width of the buffer zones, which is a portion of the main model that is important when using free-field boundary conditions.

The Abaqus analysis is conducted as a time-history analysis with a base motion defined by an accelerogram of 20 seconds' duration. We compute the Fourier amplitudes of the base motion, $U_{H}(\omega)$, and the Fourier amplitudes of the motion at the surface, $U_{0}(\omega)$, and then we evaluate the amplification function as the ratio of the two amplitudes, $A_{r}(\omega)=U_{0}(\omega) / U_{H}(\omega)$.

We use STRATA to obtain a benchmark solution for simple soil columns, and we compare the results from STRATA to those obtained from Abaqus. For simple soil columns with one layer, the STRATA solution is in fact identical to the theoretical solution represented by equation (3). The soil column specified in table 1 is used for the following calculations. The natural frequency of this soil column equals $3 \mathrm{~Hz}$ (from equation 2 ).

\begin{tabular}{|l|l|}
\hline Height, $\boldsymbol{H}[\mathrm{m}]$ & 50 \\
\hline Bulk modulus, $\boldsymbol{K}[\mathrm{MPa}]$ & 1170 \\
\hline Shear modulus, $\boldsymbol{G}[\mathrm{MPa}]$ & 540 \\
\hline Density, $\rho\left[\mathrm{kg} / \mathrm{m}^{\mathbf{3}}\right]$ & 1500 \\
\hline S-wave velocity, $\boldsymbol{V}_{\boldsymbol{s}}[\mathrm{m} / \mathrm{s}]$ & 600 \\
\hline P-wave velocity, $\boldsymbol{V}_{\boldsymbol{p}}[\mathrm{m} / \mathrm{s}]$ & 1122 \\
\hline
\end{tabular}

Table 1. Characteristics of the soil column used for the tests

Two damping levels are considered: $1 \%$ and 10\%. According to equation (3-b), the amplitudes 
should be:

$$
\begin{aligned}
& \left(A_{1 \%}{ }^{*}\right)_{\max } \cong \frac{2}{(2-1) \pi \cdot 0.01}=63.66 \\
& \left(A_{10 \%}{ }^{*}\right)_{\max } \cong \frac{2}{(2-1) \pi \cdot 0.1}=6.366
\end{aligned}
$$

The results obtained from STRATA and from Abaqus using tied boundaries and the free-field option are shown in Figure 3. The results indicate a good match between the STRATA solution and both the options of Abaqus. We note that the free-field boundary behaves as well as the tied boundary and that it agrees with the STRATA solution up to about $13 \mathrm{~Hz}$. Here we focus on frequencies up to $15 \mathrm{~Hz}$ because this is generally the range of engineering interest.
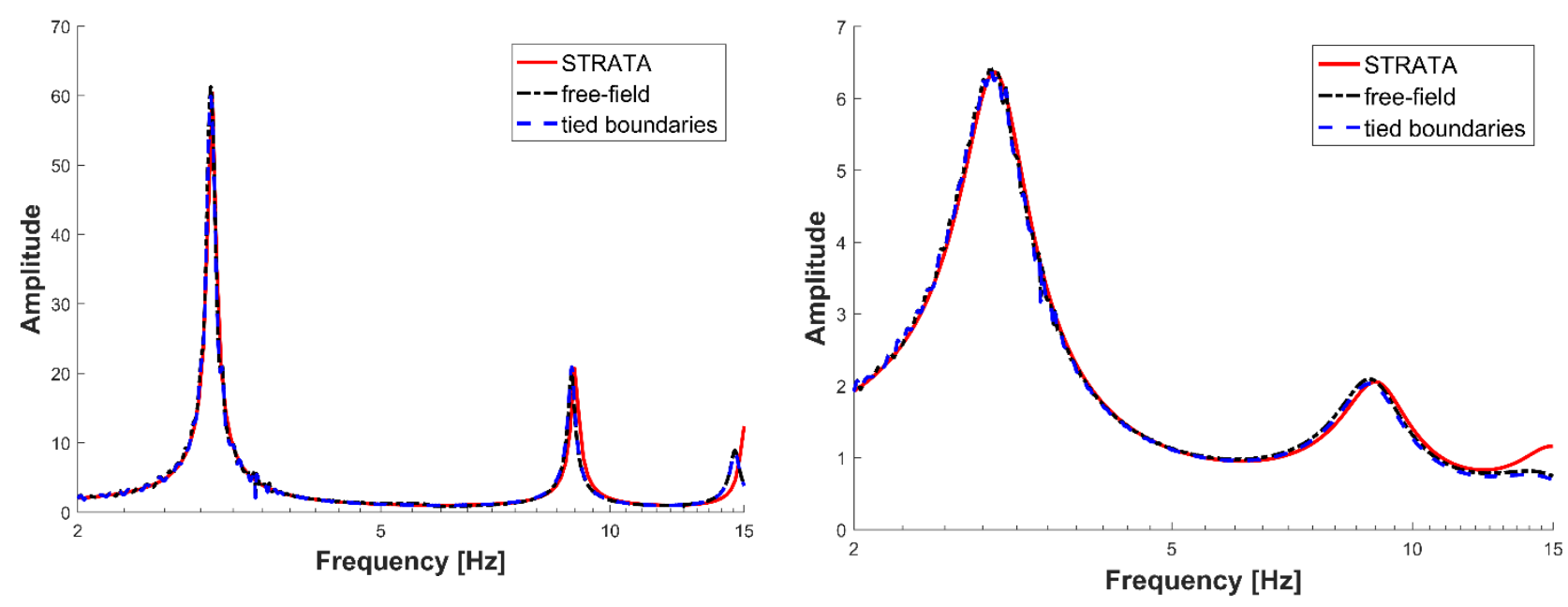

Figure 3. Comparison between Strata and Abaqus using tied boundaries and the free-field option. Left: for $1 \%$, STRATA, Free-field and Tied boundaries PGA=0.15g. Right: for $10 \%$, STRATA, Free-field and Tied boundaries PGA=0.13g

Because of its importance, we next study the influence of the mesh dimension in detail. The previous good results were obtained for a model with $V_{s}=600 \mathrm{~m} / \mathrm{s}$ and an element size of $5 \mathrm{~m}$. Now we will use the same mesh with a lower wave velocity. In this way, the consequences of violating inequality (6) will be seen. The two models listed in Table 2 are studied. 


\begin{tabular}{|l|l|l|}
\hline$H[\mathrm{~m}]$ & 50 & 50 \\
\hline $\boldsymbol{K}[\mathrm{MPa}]$ & 130 & 293 \\
\hline $\boldsymbol{G}[\mathrm{MPa}]$ & 60 & 135 \\
\hline $\boldsymbol{\rho}\left[\mathrm{kg} / \mathrm{m}^{3}\right]$ & 1500 & 1500 \\
\hline $\boldsymbol{V}_{s}[\mathrm{~m} / \mathrm{s}]$ & 200 & 300 \\
\hline $\boldsymbol{V}_{\boldsymbol{p}}[\mathrm{m} / \mathrm{s}]$ & 374 & 561 \\
\hline
\end{tabular}

Table 2. Characteristics of the two soil columns used for the tests on the mesh size

As before, the STRATA solution is compared with the results obtained using the free-field option (from Abaqus) for damping ratios of $1 \%$ and $10 \%$ in Figure 4. From the graphs, we conclude that the mesh dimension is, as expected, not adequate for the lower stiffness of the model. We also note that the level of damping has a marked influence on the result. The Abaqus solution displays considerable numerical noise for a damping ratio of $1 \%$, but this noise appears to be reduced when the damping ratio is increased to $10 \%$. However, the Abaqus solutions remain useful in terms of identifying the first two or three natural frequencies.
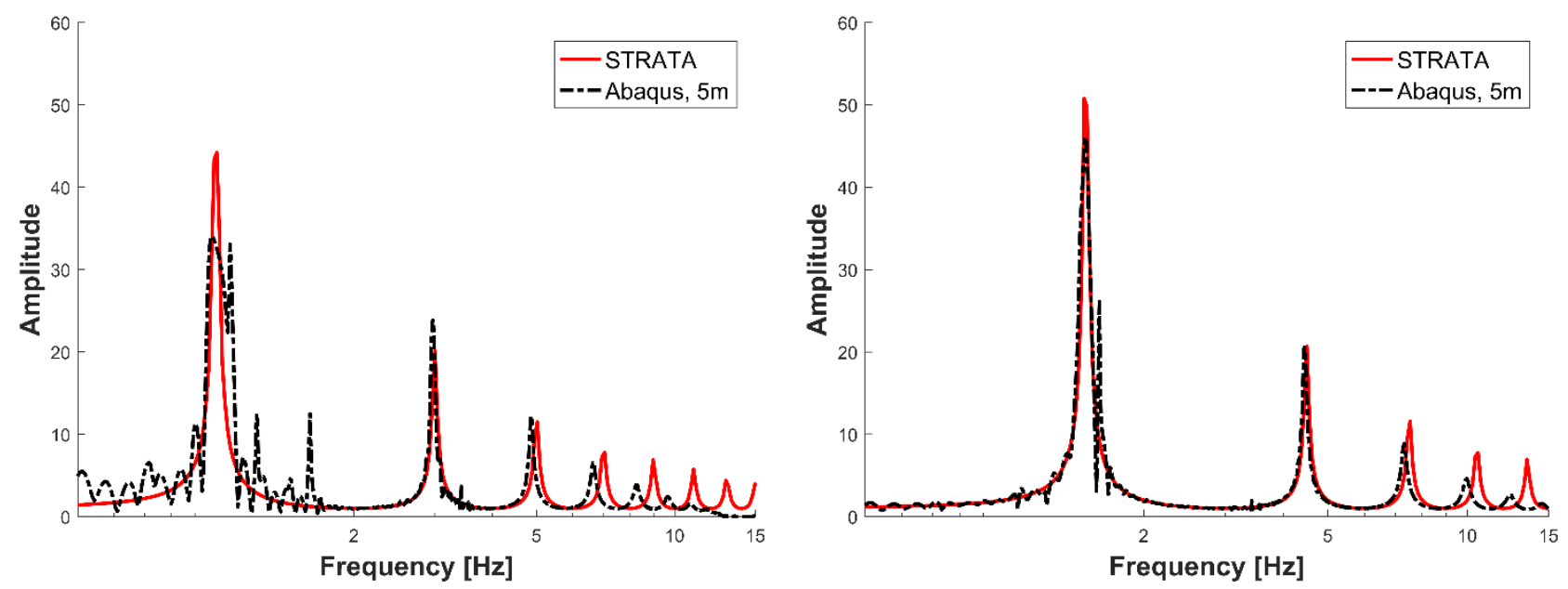

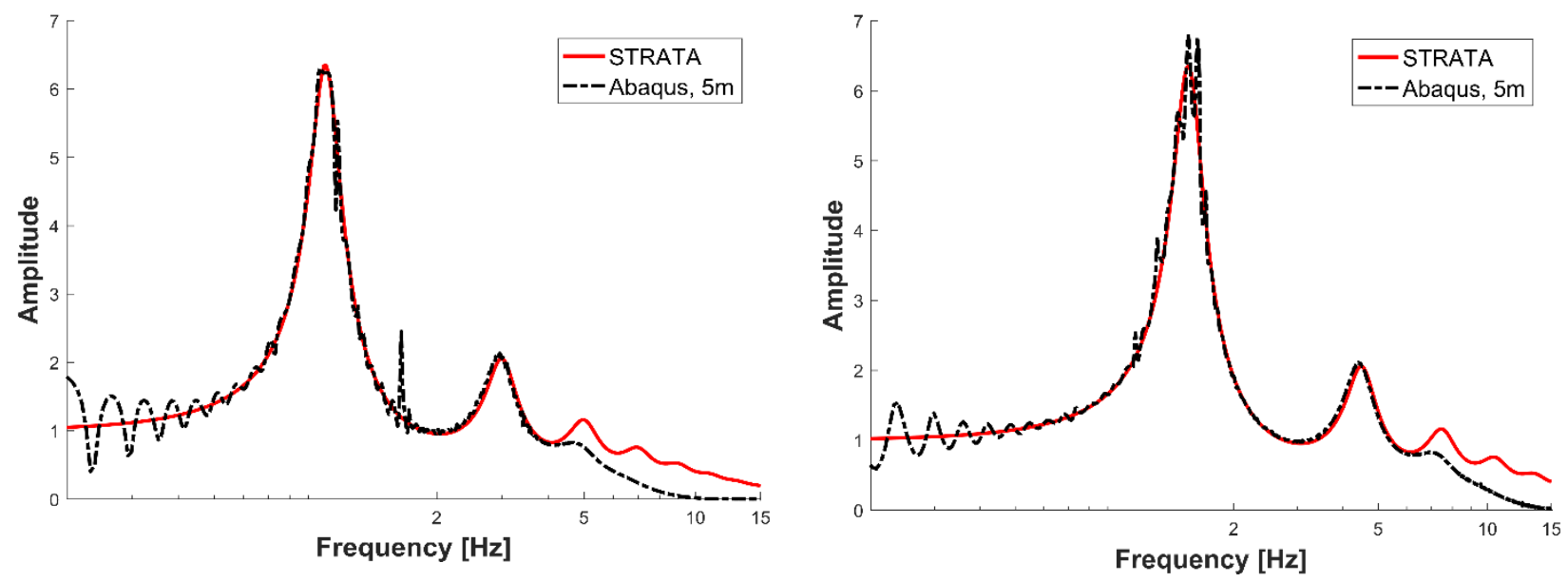

Figure 4. Comparison between STRATA and Abaqus using the free-field option. Left: Vs $=200 \mathrm{~m} / \mathrm{s}$. STRATA , $\mathrm{D}=1 \%$, PGA $=0.16 \mathrm{~g}$ Abaqus PGA=0.15g. STRATA , $\mathrm{D}=10 \%$, PGA $=0.05 \mathrm{~g}$, Abaqus , $\mathrm{D}=10 \%$, PGA=0.04g, Right: Vs=300 m/s. STRATA D=1\% PGA=0.18g, Abaqus $\mathrm{PGA}=0.17 \mathrm{~g}$, STRATA $\mathrm{D}=10 \%$ PGA $=0.08 \mathrm{~g}$, Abaqus $\mathrm{D}=10 \% \mathrm{PGA}=0.09 \mathrm{~g}$, Top: $1 \%$ damping. Bottom: $10 \%$ damping.

We consider the model with $V_{s}=300 \mathrm{~m} / \mathrm{s}$ and $1 \%$ damping in more detail. According to inequality (6) the dimensions of the elements should be smaller than $2 \mathrm{~m}$ for accurate results. Figure 5 compares the results obtained using a $2 \mathrm{~m}$ and a $1 \mathrm{~m}$ mesh. This comparison indicates that the denser the mesh, the better the match with the peak magnitude predicted by STRATA at the first natural frequency, whereas at higher frequencies a less dense mesh gives a better match to the STRATA solution.

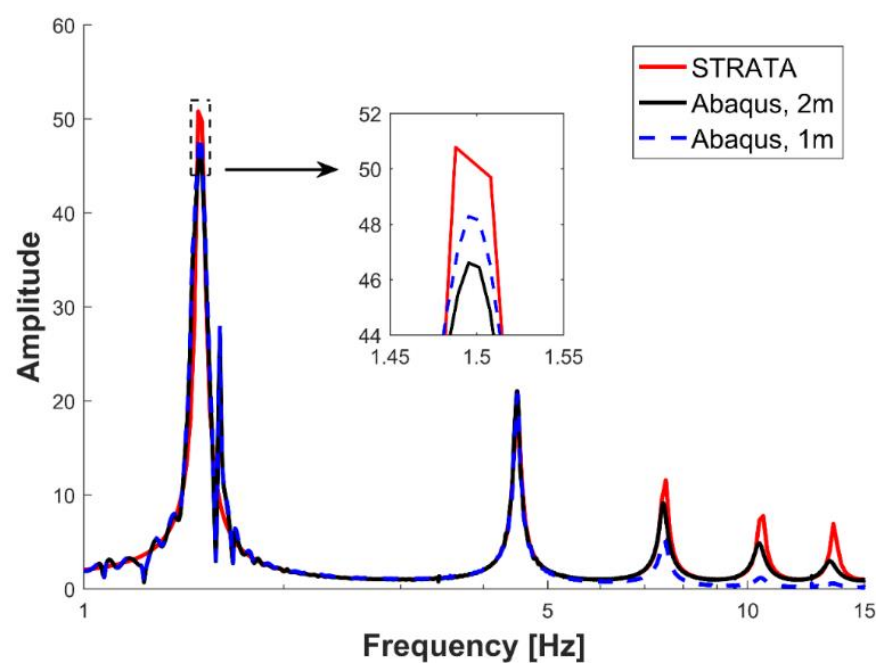


Figure 5. Comparison of the transfer function for different mesh dimensions, STRATA PGA=0.18g, Abaqus 2m PGA=0.13g, Abaqus 1m PGA=0.17g

Another important comparison to make is between results obtained using symmetric or asymmetric matrices within the free-field subroutine. Using symmetric matrices is more time efficient, but it approximates the more rigorous technique based on asymmetric matrices. Figure 6, which compares results for symmetric and asymmetric matrices, shows no differences for a soil column. Nielsen (2014) suggests that the free-field column width can be taken equal to unity for an asymmetric system as the width does not affect the results. We conducted one further test to verify this, as the right-hand figure shows.
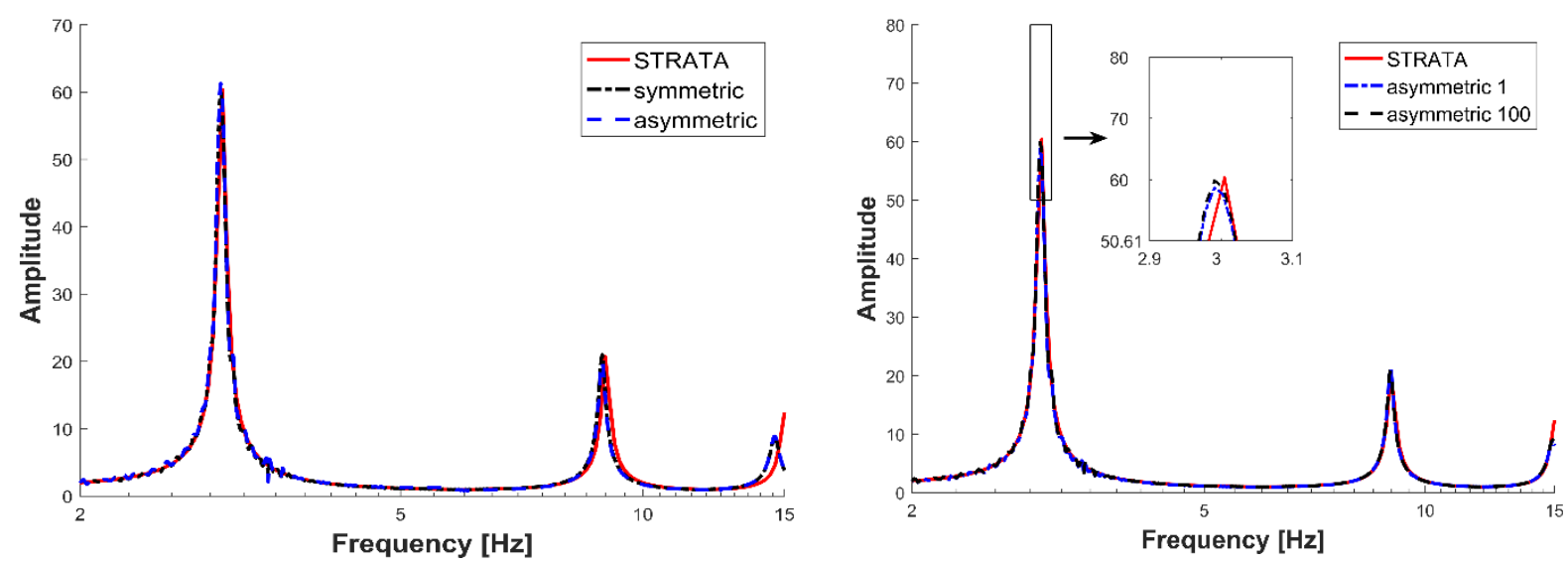

Figure $6 . \mathrm{V}_{\mathrm{s}}=600 \mathrm{~m} / \mathrm{s}$ model. On the left: comparison between symmetric matrix and asymmetric matrix; on the right: study on the influence of the width in asymmetric system, $w f f=1$ and $w f f=100$, STRATA, symmetric, asymmetric PGA=0.15g.

Another interesting point to investigate is the Rayleigh damping and its proper use, in particular to check the recommendations of Hashash and Park (2002) stated above. The model chosen for this test is the $V_{\mathrm{s}}=600 \mathrm{~m} / \mathrm{s}$ column because, owing to its stiffness, the element dimensions can be large enough for relatively rapid analyses. The fundamental frequency is equal to $3 \mathrm{~Hz}$. Several values of damping have been considered, from $0.1 \%$ (approximating an undamped model) to $10 \%$. Table 3 indicates $\alpha$ and $\beta$ for all considered frequencies and damping ratios. Examining Figure 7 leads to the following conclusions: all analyses show a good match between Abaqus results and the STRATA 
solutions for the first amplitude; solutions with $f_{1}=5 f_{0}$ and $f_{1}=7 f_{0}$ tend to overestimate the second peak as this is under-damped; solutions with $f_{1}=3 f_{0}$ present a good match for the second peak as well as the first one, whereas higher frequency peaks are over-damped.

\begin{tabular}{|c|c|c|c|c|c|c|c|c|c|}
\hline$f_{1}[\mathrm{~Hz}]$ & \multicolumn{3}{|l|}{9} & \multicolumn{3}{|l|}{15} & \multicolumn{3}{|l|}{21} \\
\hline$\xi[\%]$ & 10 & 1 & 0.1 & 10 & 1 & 0.1 & 10 & 1 & 0.1 \\
\hline$\alpha$ & 2.8 & 0.28 & 0.028 & 3.1 & 0.31 & 0.031 & 3.3 & 0.33 & 0.03 \\
\hline$\beta \times 1000$ & 2.7 & 0.27 & 0.027 & 1.7 & 0.17 & 0.017 & 1.3 & 0.13 & 0.013 \\
\hline
\end{tabular}

Table 3. $\alpha$ and $\beta$ for different values of $f_{1}$
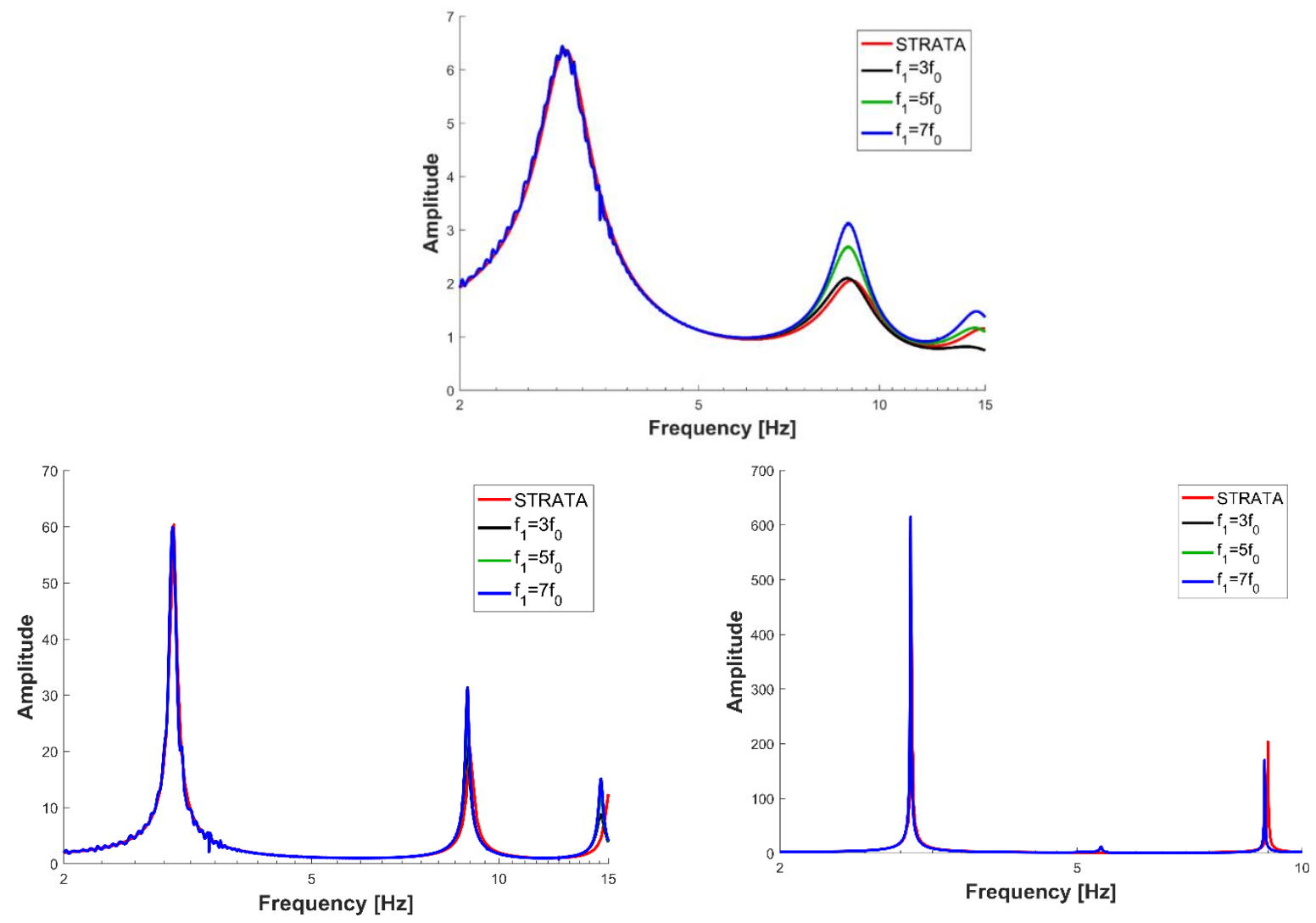

Figure 7. Comparison between transfer functions for different choices of $\alpha$ and $\beta$ depending on the frequencies targeted: $10 \%$ above, $1 \%$ below on the left (STRATA, $3 \mathrm{f}_{0}, 5 \mathrm{f}_{0}, 7 \mathrm{f}_{0}$ PGA=0.15g), $0.1 \%$ below on the right.

Finally, the influence of the width of the buffer zones width is investigated. The buffer zone must 
have a regular shape (i.e. flat, parallel layers). We consider that the width of the buffer zone should be about the same as the width of the central region of the main model, although we would recommend that the adopted buffer zone width be validated for each case. To demonstrate this, a simple example is studied here: a two-layer geometry, with an irregularity. The central region of the main model measures $50 \mathrm{~m} \times 50 \mathrm{~m}$. Two different options will be analysed (Figure 8 ): in the first one the buffer zone width is $10 \mathrm{~m}$, while in the second it is $50 \mathrm{~m}$. Results at three location points, one in the middle of each region, are studied. We will also analyse the influence of the damping ratio $(1 \%, 3 \%$ and $10 \%)$ on the results from different widths of buffer zone.

Figure 9 reports the results obtained for the central region and one of the two buffer zones. The STRATA solution for a regular site corresponding to the right-hand buffer zone is also shown for comparison. According to Seed et al. (1975), when the damping is increased the influence of geometrical irregularities on the results tends to decrease. As we expected, the influence of buffer zone width is larger for lower levels of damping. Moreover, it would seem that the model with $10 \mathrm{~m}$ width of buffer zone does not capture the correct peak associated with the first natural frequency in the buffer zone. This difference becomes less important as the damping level increases.

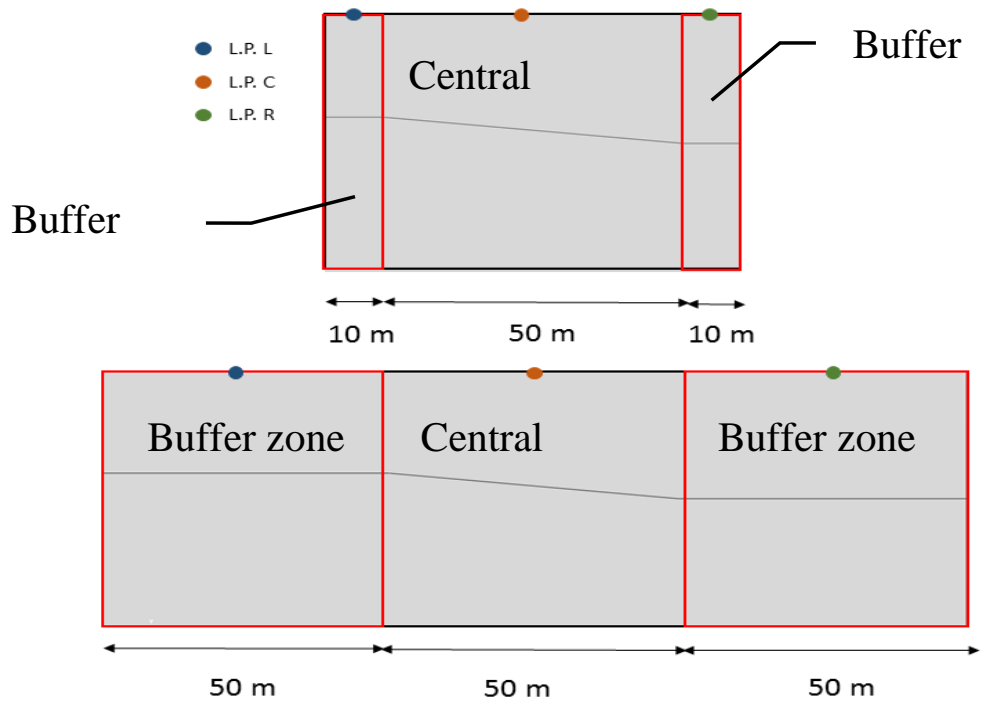

Figure 8. The domains and output locations considered for the buffer zone comparisons. 

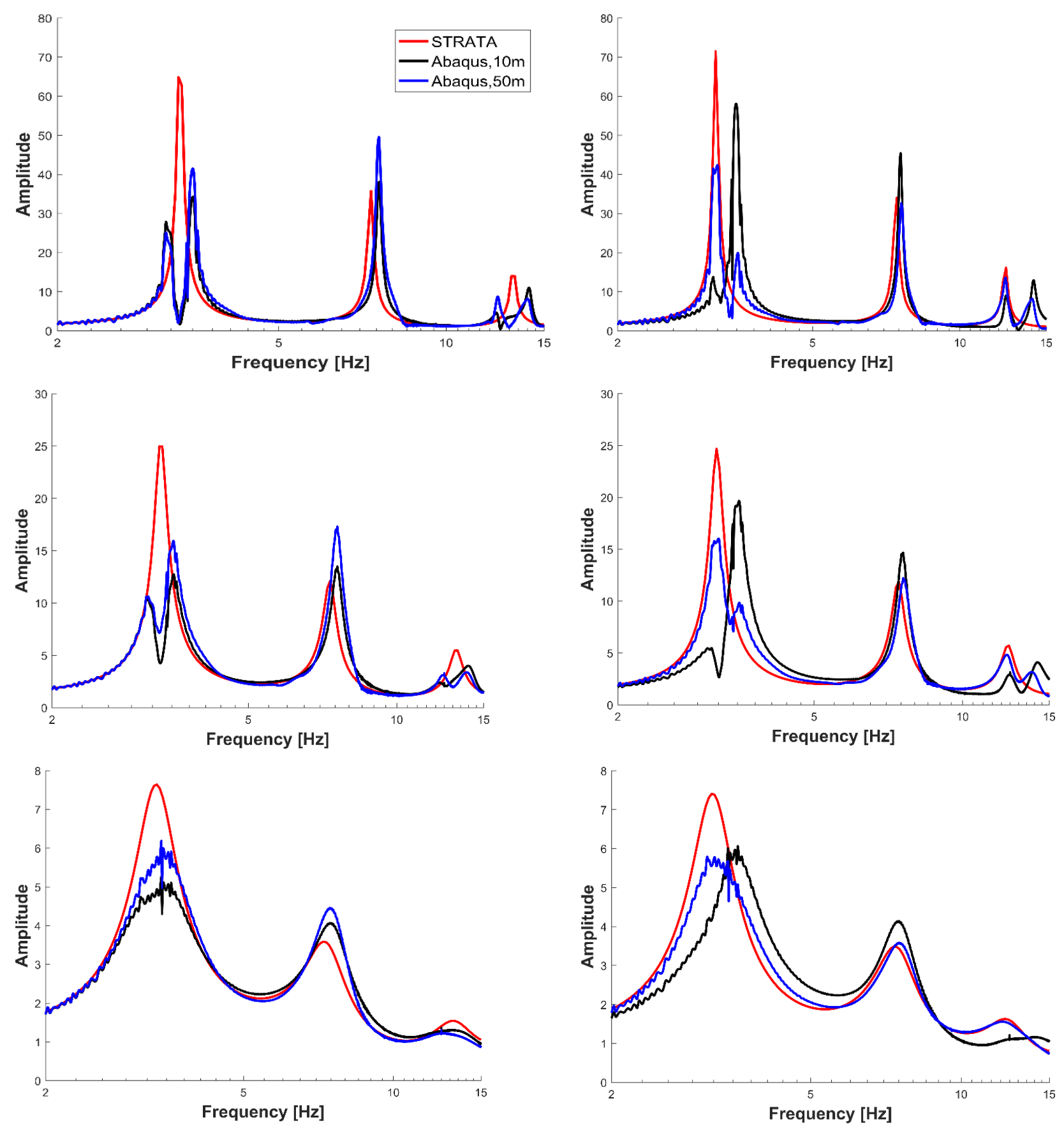

Figure 9. Comparisons in transfer functions for different buffer zone widths and damping ratios. Top: $1 \%$. Middle: 3\%. Bottom: 10\%. Left: Centre. STRATA d=1\% :PGA=0.34g, Abaqus 50m PGA $=0.40 \mathrm{~g}$, Abaqus $10 \mathrm{~m}$ PGA=0.37g. Right: Right-hand buffer zone. STRATA=0.35g Abaqus 50m PGA $=0.34 \mathrm{~g}$, Abaqus $10 \mathrm{~m}=0.41 \mathrm{~g}$, Left: Centre. STRATA d=3\% :PGA=0.32g, Abaqus 50m PGA $=0.34 \mathrm{~g}$ Abaqus 10m PGA=0.34g Right: Right-hand buffer zone. STRATA=0.30g Abaqus 50m PGA $=0.31 \mathrm{~g}$ Abaqus $10 \mathrm{~m}=0.34 \mathrm{~g}$, STRATA d=10\% $: \mathrm{PGA}=0.25 \mathrm{~g}$, Abaqus 50m PGA $=0.25 \mathrm{~g}$, Abaqus 
10m PGA=0.26g. Right: Right-hand buffer zone. STRATA=0.23g Abaqus 50m PGA=0.23g, Abaqus $10 \mathrm{~m}=0.25 \mathrm{~g}$,

\section{Testing FLAC3D}

FLAC3D is based on the finite-difference method using an explicit time integration scheme. An interesting comparison between implicit and explicit time integration schemes can be found in Andreotti and Lai (2017a, b). In contrast to Abaqus, FLAC3D has the free-field boundary condition implemented. To use them in this program, we need to append a buffer zone to any irregular geometry so as to provide a smooth transition to the regular geometry that is the basis of the freefield model. As rule of thumb, we consider that the width of a buffer zone should be equal to the width of the main model.

We also note that within an explicit integration scheme the dynamic time step changes according to the level of damping. The higher the damping, the smaller the time-step becomes, which means that analysis will take longer.

Above Abaqus is tested for simple geometries, i.e. a single layer soil column. To check its behaviour for more complex situations, we need to compare it to another software that is also capable of 2D SRA. 2D models can be developed in FLAC3D by developing a 3D model with a unit width in the breath direction.

A test of the Rayleigh damping was made for FLAC3D because, as previously mentioned, this software uses a simplified formula using only a single control frequency, $f_{\min }$. We test the influence of the control frequency choice for a simple model (Table 3, Figure 10). The results show that by fixing the control frequency as the natural frequency, $f_{\min }=f_{0}$, the first amplitude is overestimated, while $f_{\min }=f_{1}$ as control frequency tends to underestimate the first peak, but has a good match for the second and third peaks. Choosing $f_{\min }=f_{\mathrm{a}}=\left(f_{0}+f_{1}\right) / 2$ appears as the best comprise between the 
two, because overall it leads to the best fit.

\begin{tabular}{|l|l|}
\hline$K[\mathrm{MPa}]$ & 1340 \\
\hline $\boldsymbol{G}[\mathrm{MPa}]$ & 446 \\
\hline$\rho\left[\mathrm{kg} / \mathrm{m}^{3}\right]$ & 2200 \\
\hline $\boldsymbol{V}_{\boldsymbol{s}}[\mathrm{m} / \mathrm{s}]$ & 450 \\
\hline $\boldsymbol{H}[\mathrm{m}]$ & 50 \\
\hline$f_{0}[\mathrm{~Hz}]$ & 2.25 \\
\hline$f_{1}[\mathrm{~Hz}]$ & 6.75 \\
\hline$f_{\mathrm{a}}[\mathrm{Hz}]$ & 4.5 \\
\hline$\xi[\%]$ & 1 \\
\hline
\end{tabular}

Table 3. Characteristics of the model in FLAC3D

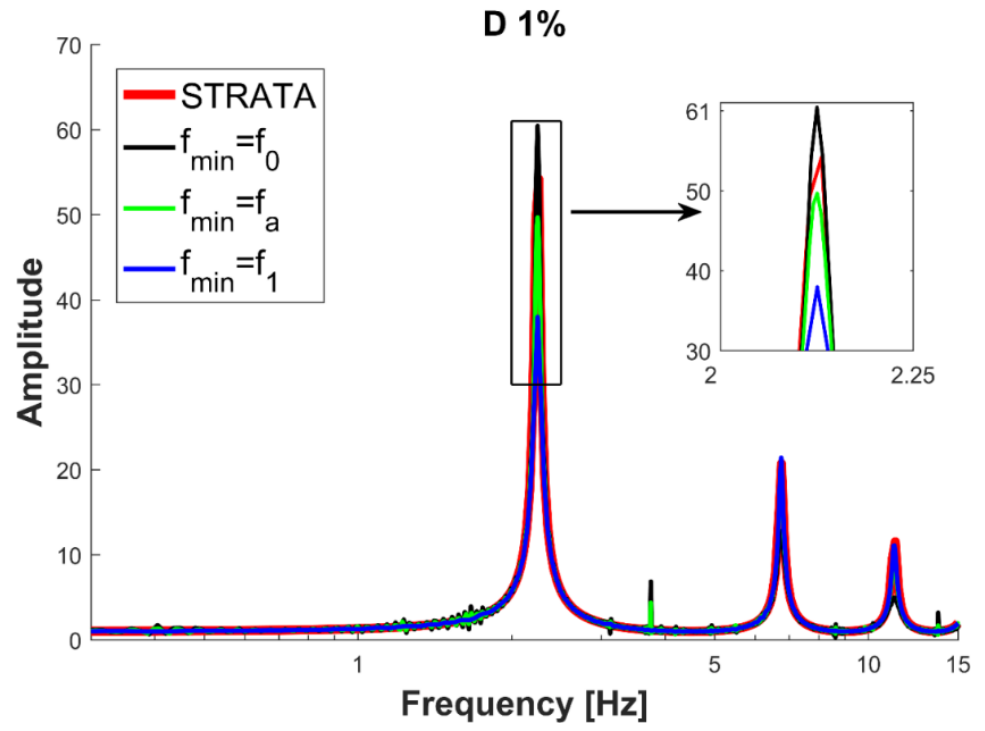

Figure 10. The influence of the control frequency in FLAC3D on the transfer function. STRATA, $\mathrm{f}_{0}, \mathrm{f}_{\mathrm{a}} \mathrm{PGA}=0.52 \mathrm{~g}, \mathrm{f}_{1} \mathrm{PGA}=0.51 \mathrm{~g}$ 


\subsection{Comparing Abaqus and FLAC3D}

In this section a comparison between the results from Abaqus and FLAC3D for an irregular 2D geometry is made. Viscously damped elastic analyses for a stratigraphy consisting of three layers (Table 5, Figure 1) are made.

\begin{tabular}{|l|l|}
\hline Total width [m] & 120 \\
\hline Buffer zone width [m] & 40 \\
\hline Central region width [m] & 40 \\
\hline H1 left [m] & 10 \\
\hline H2 left [m] & 20 \\
\hline H3 left [m] & 30 \\
\hline H1 right [m] & 13 \\
\hline H2 right [m] & 20 \\
\hline H3 right [m] & 27 \\
\hline
\end{tabular}

Table 5. Geometries of the three dipping layers considered

The properties of the model are reported in Table 6 .

\begin{tabular}{|l|l|l|l|}
\cline { 2 - 4 } \multicolumn{1}{l|}{} & LAYER1 & LAYER2 & LAYER3 \\
\hline $\boldsymbol{H}[\mathrm{m}](\mathbf{c e n t r e})$ & 11.5 & 20 & 28.5 \\
\hline $\boldsymbol{G}[\mathrm{MPa}]$ & 298 & 1500 & 1800 \\
\hline $\boldsymbol{\rho}\left[\mathrm{kg} / \mathbf{m}^{3}\right]$ & 170 & 500 & 700 \\
\hline $\boldsymbol{V}_{\boldsymbol{s}}[\mathrm{m} / \mathbf{s}]$ & 1800 & 1900 & 2200 \\
\hline$f_{\mathbf{0}}[\mathrm{Hz}]$ & 307 & 513 & 564 \\
\hline$f_{1}[\mathrm{~Hz}]$ & 6.6 & 3.2 & 1.9 \\
\hline $\boldsymbol{\alpha}$ & 20 & 9.8 & 5.9 \\
\hline
\end{tabular}




\begin{tabular}{|l|l|l|l|}
\hline$\beta \times 1000$ & 0.119 & 0.24 & 0.404 \\
\hline$f_{\text {min }}[\mathrm{Hz}]$ & 2.6 & & \\
\hline
\end{tabular}

Table 6. Properties of the model with three dipping layers

Results for three locations: one in the left part of the model, one in the centre and one in the right part of the model are shown in Figure 11. These comparisons show that both Abaqus and FLAC3D produce similar transfer functions. In particular, the same resonant frequencies are identified, although the maximum amplitudes differ because of their different Rayleigh damping formulations. Specifically, FLAC3D uses a single control frequency, which means there is less control on the frequency dependence of the damping.
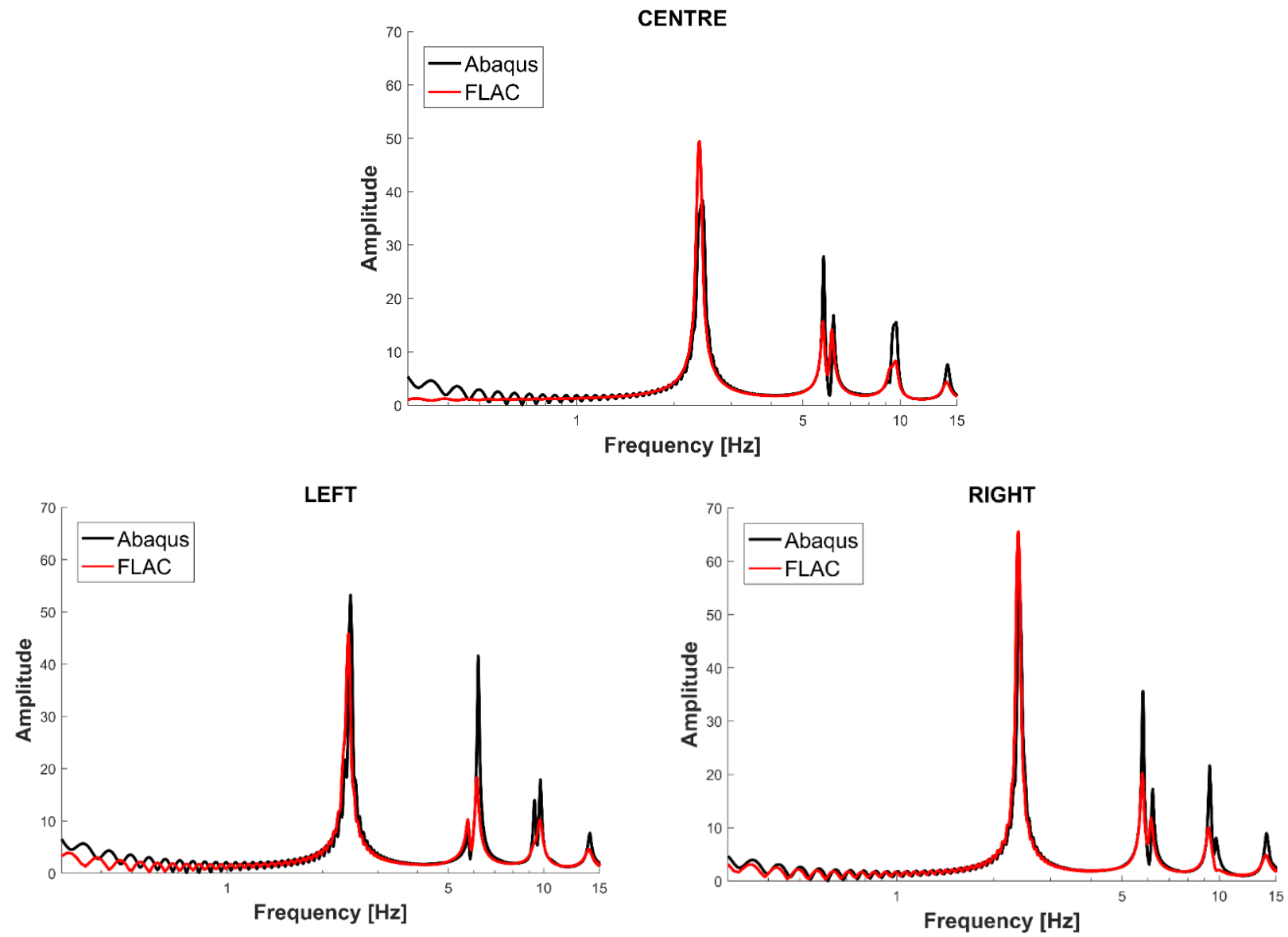

Figure 11. Abaqus and FLAC3D transfer functions for the three dipping layers model, Abaqus centre PGA $=0.045 \mathrm{~g}$, FLAC PGA $=0.05 \mathrm{~g}$ 
These results suggest Abaqus can simulate free-field boundary conditions in a satisfactory way. Moreover, we found that Abaqus is faster for linear-elastic analyses than FLAC3D.

\section{Conclusions}

In this work, a finite-element code (Abaqus) has been tested for 1D and 2D dynamic site response analyses. As a general-purpose FE application, Abaqus does not have the specialised boundary conditions required for this type of analysis. Abaqus does provide infinite elements for both static and dynamic analysis, but they are not optimal for SRA. The free-field boundary conditions as developed by Nielsen $(2006,2014)$ are more appropriate for SRA of irregular sites. The free-field boundaries were tested in different configurations, and satisfactory results were found. Specifically, we found that:

- models with free-field boundaries provide the correct solutions for regular sites (by comparison with tied boundaries and STRATA solutions);

- choosing the appropriate element dimensions is crucial, especially for low values of damping ratio;

- the free-field boundaries can be used with symmetric matrices which permits faster solutions; and

- the Hashash and Park (2002) recommendations for the evaluation of control frequencies for Rayleigh damping are suitable.

Having clarified these aspects, we tested the importance of the buffer zone for models with irregularities. As a rule of thumb, the width of the buffer zone should be about the same as the width of the central region of the main model. However, we recommend that the adopted buffer zone width be validated for each case, for example by increasing the width until the output time history (or response spectrum) in the region of interest converges to a stable value. 
Finally, we compared the Abaqus results with results from the finite-difference program, FLAC3D, where the free-field boundary condition is already implemented. Results have shown that the Abaqus implementation works properly when the guidance provided in this article is followed.

\section{Acknowledgements}

We would like to thank the anonymous reviewers for their useful comments on a previous version of this technical note.

\section{Bibliography}

Abaqus, 6.13 @ 2002-2018 Dassault Systèmes - All rights reserved

Andreotti G, Lai C.G., (2017). A nonlinear constitutive model for beam elements with cyclic degradation and damage assessment for advanced dynamic analyses of geotechnical problems. Part I: theoretical formulation. Bulletin of Earthquake Engineering 15(7): 2785-2801. DOI: $\underline{10.1007 / \mathrm{s} 10518-017-0090-1}$

Andreotti G, Lai C.G., (2017). A nonlinear constitutive model for beam elements with cyclic degradation and damage assessment for advanced dynamic analyses of geotechnical problems. Part II: validation and application to a dynamic soil-structure interaction problem. Bulletin of Earthquake Engineering 15(7):2803-2825. DOI: 10.1007/s10518-017-0091-0. Bard, P.-Y., Bouchon M., 1980,a “The Seismic Response of Sediment- Filled Valleys . Part 1. The Case of Incident SH Waves.” Bulletin of the Seismological Society of America 70 (4): 1263-1286.

Bard, P.-Y., Bouchon M., 1980,b “The Seismic Response of Sediment- Filled Valleys . Part 2. The Case of Incident P and SV Waves." Bulletin of the Seismological Society of America 70 (5): 1921-1941.

Bard, P.-Y., Bouchon M., 1985. "The Two-Dimensional Resonance of Sediment-Filled Valleys.” Bulletin of the Seismological Society of America 75(2):519-41.

Bathe K.-J., Wilson E. L., 1976, "Numerical methods in finite element analysis", Englewood Cliffs, NJ: Prentice-Hall.

Bonilla, L.F., 2000. NOAH: Users Manual, Institute for Crustal Studies, University of California, Santa Barbara

Chávez-García, F.J., Faccioli E., 2000. "Complex Site Effects and Building Codes: Making the Leap.” Journal of Seismology 4(1):23-40. 
Chopra, A.K., 1995. Dynamics of Structures: Theory and Applications to Earthquake Engineering. Prentice-Hall, New Jersey.

Hashash Y.M.A., Park D., 2002. "Viscous damping formulation and high frequency motion propagation in non-linear site response analysis", Soil Dynamics and Earthquake Engineering 22 (2002) 611-624

Itasca Consulting Group Inc. FLAC, 2012, Itasca Consulting Group Inc. FLAC 3DFast Lagrangian Analysis of Continua: User's Manual, Minneapolis.

Kaklamanos J., Bradley B. A., Thompson E. M., Baise L. G. 2013. Critical Parameters affecting Bias and variability in Site-Response analysis using Kik-net downhole array data, Bulletin of the Seismological Society of America 103(3): 1733-1749, https://doi.org/10.1785/0120120166

Kaklamanos J., Baise L. G., Thompson E. M., Dorfmann L., 2015. Comparison of 1D linear, equivalent-linear and nonlinear site response models at six KiK-net validation sites, Soil Dynamics and Earthquake Engineering, Volume 69, 207-219, https://doi.org/10.1016/j.soildyn.2014.10.016

Kimball A.L., Lovell D.F., 1927. Internal friction in solids, Physics Review 30 , 948

Kottke, A. R., \& Rathje, E. M., 2008. Technical Manual for Strata, PEER Report 2008/10, Pacific Earthquake Engineering Research Center College of Engineering, University of California, Berkeley.

Kramer, S. L., 1996, “Geotechnical earthquake engineering”, Prentice Hall, Upper Saddle River, N.J

Kuhlemeyer, R.L. and Lysmer. J., 1973. Finite Element Method Accuracy for Wave Propagation Problems. Journal of the Soil Dynamics Division, 99, 421-427.

Lysmer, J. \& Kuhlemeyer, R.L., 1969. Finite dynamic model for infinite media, Proceedings of the ASCE - Journal of the Engineering Mechanics Division, 95, no. EM4, 859-876

Luzi L., Pacor F., Puglia R., 2017. Italian Accelerometric Archive v2.3. Istituto Nazionale di Geofisica e Vulcanologia, Dipartimento della protezione Civile Nazionale, doi:10.13127/ITACA.2.3.

Nielsen, A. H., 2006. Absorbing Boundary Conditions for Seismic Analysis in ABAQUS. ABAQUS Users' Conference 359-76.

Nielsen, A. H., 2014. Towards a Complete Framework for Seismic Analysis in Abaqus, Proceedings of the ICE - Engineering and Computational Mechanics 167(1):3-12.

Rayleigh J.W.S., Lindsay R.B., 1945. The theory of sound, 1st American ed. New York: Dover Publications. 
Schnabel, P.B., Lysmer, J. and Seed H.B., 1972. Shake: a computer program for earthquake response analysis of horizontally layered sites, Report EERC 72-12, Earthquake Engineering Research Center, University of California, Berkeley

Schnabel P.B., Lysmer J., Seed H.B., 1991. SHAKE-91: Equivalent Linear Seismic Response Analysis of Horizontally Layered Soil Deposits

Seed, H. B., Martin P. P., Lysmer J., 1975. The Generation and Dissipation of Pore Water Pressures During Soil Liquefaction, University of California, Berkeley, Earthquake Engineering Research Center, NSF Report PB-252 648

Stewart, J. P., K. Afshari, and Y. M. A. Hashash, 2014. Guidelines for performing hazard-consistent one-dimensional ground response analysis for ground motion prediction, PEER Report No. 2014/16, Pacific Earthquake Engineering Research Center, UC Berkeley, CA.

Stewart, J. P., K. Afshari, and C. A. Goulet, 2017. Non-Ergodic site response in seismic hazard analysis, Earthquake Spectra, 33, 1385-1414.

Thompson, E. M., L. G. Baise, Y. Tanaka, and R. E. Kayen, 2012. A taxonomy of site response complexity, Soil Dyn. Earthq. Eng., 41, 32-43.

Vidale J., Helmberger D. V., 1988, Elastic finite difference of the 1971 San Fernando earthquake, Bulletin of the Seismological Society of America, Vol. 78, No 1, pp. 122-141

Volpini C., Douglas J., 2018. An accessible approach for the site response analysis of quasihorizontal layered deposits, Bulletin of Earthquake Engineering, https://doi.org/10.1007/s10518-018-0488-4 (in press)

Wolf, J.P., 1994. Foundation Vibration Analysis Using Simple Physical Models, Prentice Hall.

Wolf, J.P., 1988. Soil-Structure Interaction Analysis in Time Domain. Prentice Hall.

Zienkiewicz, O.C., N. Bicanic and F.Q. Shen, 1989 Earthquake input definition and the transmitting boundary conditions, Proceedings Advances in Computational Nonlinear Mechanics I, pp. 109- 138, Springer-Verlag 